\title{
Blow-assisted multi-jet electrospinning of poly-L-lactic acid nanofibers
}

\author{
Michał Wojasiński ${ }^{1}$ [D $\cdot$ Jakub Golawski $^{1} \cdot$ Tomasz Ciach $^{1,2}$
}

Received: 31 October 2016 / Accepted: 10 April 2017 /Published online: 19 April 2017

(C) The Author(s) 2017. This article is an open access publication

\begin{abstract}
Regardless the low production rate, electrospinning remains the attractive technique for the nanofibers production in various fields. Thus, the development of a multi-jet technologies for electrospinning gives an opportunity to scale up and increase throughput of the fibers production. However, the multi-jet electrospinning technologies exhibit one major drawback- electrostatic mutual jet repulsion issue. In present research, we propose air blow-assisted multi-jet electrospinning system allowing production of nanofibers with yield, at least, tenfold higher than single jet electrospinning. The system produces nanofibers in two modes: multi-jet electrospinning and blow-assisted multi-jet electrospinning. In case of the latter, the application of sheath air stream allows the system to overcome the electrostatic mutual repulsion issue. These lead to the reduction of deviation of the polymer solution jets, the reduction of instabilities of the jets and the improvement of the control of the nanofibers deposition. Nanofibers morphology and size were investigated based on the scanning electron microscope micrographs. The comparison of the two modes shows changes in nanofibers morphology from beaded structure to fine nanofibers, and the slight increase in fiber mean size when the blowing assistance was applied to the process.
\end{abstract}

Michał Wojasiński

m.wojasinski@ichip.pw.edu.pl

1 BioMedical Engineering Laboratory, Department of Biotechnology and Bioprocess Engineering, Faculty of Chemical and Process Engineering, Warsaw University of Technology, Waryńskiego 1, 00-645 Warsaw, Poland

2 CEZAMAT PW, Polna 50, 00-644 Warsaw, Poland
Keywords Nanofibers · Poly-L-lactic acid (PLLA) $\cdot$ Multi-jet electrospinning $\cdot$ Blow-assisted electrospinning

\section{Introduction}

The main limitation of electrospinning process is a low nanofibers production rate. Since 1934, when Fromhals introduced the electrospinning to wider public by submitting a patent application for the technique, researchers have been investigating possibilities of its' industrial usage [1]. On the laboratory scale, the electrospinning allows to produce ultrafine nanofibers with rates sufficient enough to conduct experiments. It means that using polymer solution feed rate in the range from about $0.2 \mathrm{ml} \cdot \mathrm{h}^{-1}$ to about $2 \mathrm{ml} \cdot \mathrm{h}^{-1}$ in a single-jet electrospinning system fibers can be produced with a rate from about 0.01 to about $0.1 \mathrm{~g} \cdot \mathrm{h}^{-1}[2,3]$. Other obstacles with industrial application of electrospinning are the high voltage requirement and humidity influence of the fibers properties. Even though the industrial application of the electrospinning is limited, nanofibrous structures generated by this technology gathered attention of specialists from various fields, for instance filtration $[4,5]$, chemical catalysis $[6,7]$, electronics and energy storage $[8,9]$, tissue engineering [10-12], and the like.

To overcome the low throughput of electrospinning, and provide large scale process for abovementioned specialists, various approaches have been reported to scale up nanofiber production. These approaches might be named as multi-jet techniques. Zheng et al. summarized these approaches to electrospinning scale up in three categories [13]: multineedle electrospinning [14, 15], multihole electrospinning [16, 17], and free surface electrospinning $[18,19]$. Even though above approaches allow improvement in nanofibers production rate by electrospinning, there is an important issue with multi-jet 
electrospinning - the deviation of the angle of the jets form the axis of the electrospinning system caused by mutual electrostatic repulsion. The use of more than one nozzle for polymer solution supply in multi-jet electrospinning causes the electric field deviation that leads to jets repulsion and angle deviation of the jets. The deviation of multi-jet electrospinning jets also causes instability problems such as dripping of polymer solution and fiber collection difficulties [3]. Working on single-jet electrospinning process, Deitzel et al. proposed application of additional rings to stabilize the electric field [20], and this approach inspired variety of configurations for multi-jet electrospinning to modify and control the process. Kim et al. introduced multi-jet electrospinning system with additional cylindrical electrode surrounding the nozzles system [21]. Another modification by additional electrodes in multi-jet process was an auxiliary electrode. Placed in parallel to the collector, either supporting the nozzles [15, 22], or working as additional collector [23], the auxiliary electrode controls the electric field uniformity in the multi-jet electrospinning process. Tomaszewski and Szadkowski, and Yang et al. suggested that appropriate nozzles distribution in multi-jet electrospinning system facilitates collecting process of nanofibers [24, 25]. In their review paper, Ramakrishnan et al. described another approach to multi-jet needleless electrospinning called bubble electrospinning [26]. In this process, carrier gas is pushed through the polymer solution forming bubbles. When a bubble bursts at the surface of the polymer solution, the polymer jet is formed in electrostatic field. Another approach to overcome the instabilities of polymer jet in electric field for the single-jet electrospinning also involves usage of the gas and includes application of high speed air stream. Several groups used this approach to control the production and the collecting process in a simple single-jet electrospinning system. This approach was also used to improve the nanofibrous product quality [27-29]. Even though, the last two abovementioned approaches are not specific for overcoming the mutual repulsion effect, the application of, so called, assistance of sheath gas was inspired and reported to be used in multi-jet electrospinning systems [30]. Process presented by $\mathrm{Yu}$ et al. involves the application of air stream in the way similar to the solution blow spinning process - another method for nanofibers production [31] - where each spinneret is built in a way allowing the use of gas stream individually. This approach gives a control over each polymer solution jet separately, but leaves the problem of non-uniform collection of the nanofibers mat on the surface of the collector.

Modifications of multi-jet electrospinning process presented above are based on both phenomenological and numerical approaches to solving the problem of mutual jet repulsion in the process. Zheng et al. offered more detailed description of this problem. Testing multihole and multineedle systems for electrospinning, they discovered that the factors for good control of the collecting process of nanofibers are the length of the straight jet and the size of the envelope cones of the outer jets. Also, according to the results of Zheng et al., the outer jets angle deviates more and create larger envelope cones in multineedle electrospinning system [3].

Our previous study shows that application of air stream in nanofibers producing processes may play a crucial role, especially when it is the only source of a driving force for the process [32]. In present work, we introduce the air blowing assistance to multi-jet electrospinning process. Application of the multi-jet electrospinning system was intended to improve the electrospinning throughput. The aim of present work is to present a new way to overcome electrostatic mutual repulsion of the polymer jets by the application of sheath gas surrounding multi-jet electrospinning spinneret using specifically designed system. The effect of the reduction of the repulsive interactions of polymer jets was investigated. This effect was measured by the size of collection area of the nanofibrous mat on the surface of the collector, and comparison of the products obtained in two modes multi-jet electrospinning and blowassisted multi-jet electrospinning was made. What is more, we investigated how the modification of the multi-jet electrospinning process by blowing assistance influences the nanofibers quality - fibers morphology and fibers mean size.

\section{Materials and methods}

\section{Blow-assisted multi-jet electrospinning system}

The multi-jet electrospinning (MES) technology with assistance of blowing air was developed, and the spinneret of blow-assisted multi-jet electrospinning (BMES) system is shown in Fig. 1. The BMES system consisted of the following components. Polymer solution was delivered to the system by syringe pump (Ascor, Poland, Fig. 1d-1) using $20 \mathrm{ml}$ syringe (BD Plastipak, USA) to the polymer solution chamber in multi-jet system (Fig. 1a). The multi-jet electrospinning spinneret consisted eight polymer nozzles - inner diameter of $0.8 \mathrm{~mm}$ - placed on the electrode (Fig. 1a-1). The aluminum ring with outer diameter of $45 \mathrm{~mm}$ and inner diameter of $20 \mathrm{~mm}$ was used as an electrode (Fig. 1a-2). The polymer nozzles were arranged on the electrode on the circumference of a concentric circle having diameter of $32 \mathrm{~mm}$, at the apexes of the octagon inscribed in that circle. The electrode was placed on the polymer solution chamber (Fig. 1a-4). Further, the system consisted an air duct and mount (Fig. 1a-5 and a-6, respectively) placed onto the support (Fig. 1a-7). Compressed air, with controlled pressure, was delivered by the air pump (HydrovaneHV01, UK) through the inner and outer air nozzles (Fig. 1c). The inner air nozzle had the diameter of $20 \mathrm{~mm}$, and the outer air nozzle $-120 \mathrm{~mm}$. At a distance of $60 \mathrm{~mm}$ (working distance) over the polymer solution nozzles the collecting aluminum plate was mounted. The electrode and 
Fig. 1 Blow-assisted multi-jet electrospinning system (BMES); (a) multi-jet electrospinning (MES) spinneret: 1 - polymer nozzles, 2 - electrode,

3 - electrode support, 4 - polymer solution chamber, 5 - air duct, 6 - mount, 7 - base; (b) MES axial cross-section; (c) BMES air flow schematic - inside and outside; (d) schematic of MES and BMES system: 1 - syringe pump (polymer solution source), 2 - spinning chamber, 3 - high voltage power supply (air source, connected to base, is not shown)

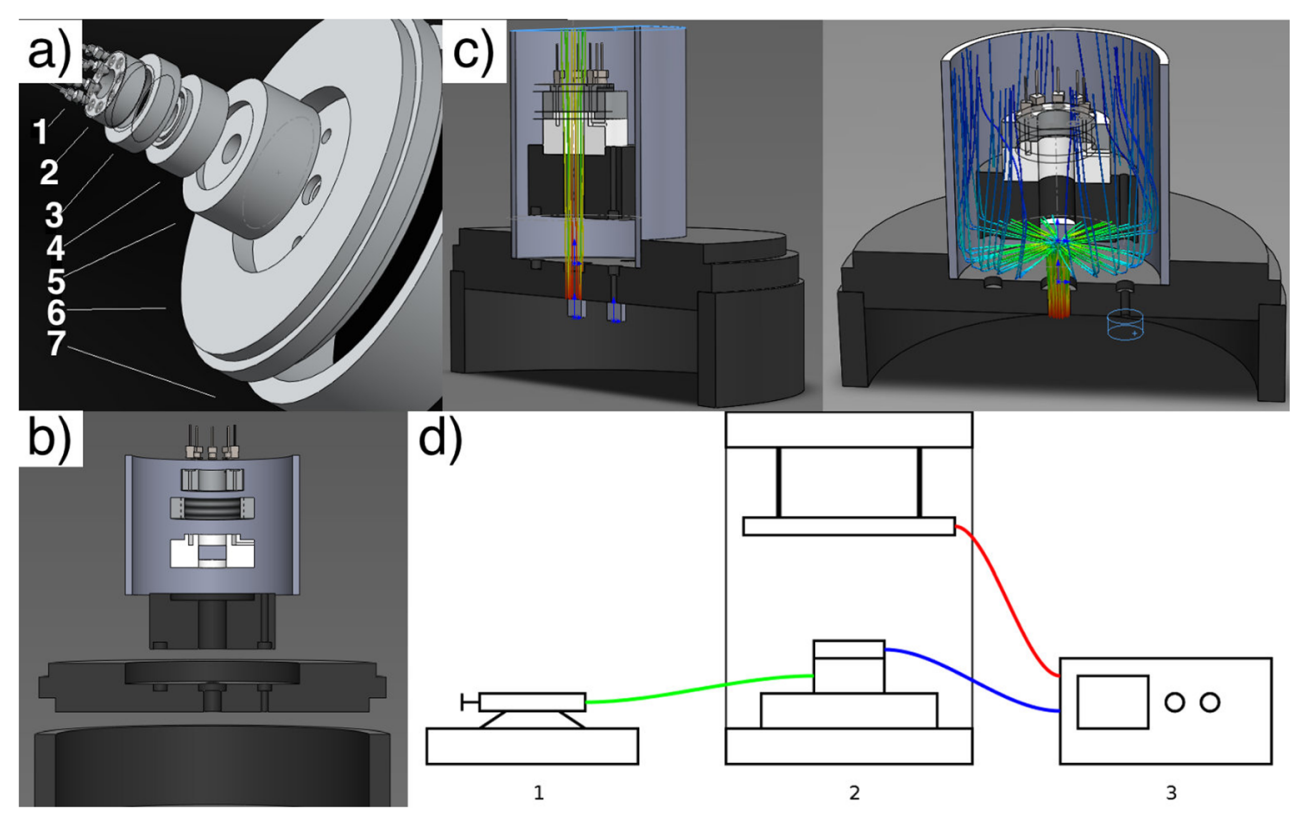

the collecting plate were connected to the high voltage power supply, to the positive connector and the grounding, respectively. The Electrostatic Generator ET-100/F (JJ Serwis, Poland, Fig. 1d-3) was used as a high voltage power supply to provide voltages in the range from 0 to $25 \mathrm{kV}$.

\section{Polymer solutions}

Poly-L-lactic acid (PLLA) with weight-average molecular weight about 200 kDa (Biomer L9000, Krailing, Germany) was used to prepare solutions for both, multi-jet electrospinning (MES) and blow-assisted multi-jet electrospinning (BMES) processes. Mixture of dichloromethane and N,N-dimethylformamide $(9: 1, v / v)$ was used as a solvent (both analytical grade, Carlo Erba Reagents, Italy). The PLLA solutions of mass concentration of 4,6 and $8 \% \mathrm{w} / \mathrm{w}$, were prepared $24 \mathrm{~h}$ before the experiments. Preparation of the polymer solutions was conducted in the room temperature, and relative humidity was in a range between 40 to $55 \%$, conditions corresponding to those of MES and BMES processes. Zero-shear rate viscosity of the prepared solutions was determined using Brookfield DV-III+ rheometer (Brookfiled, Inc., USA). Resulting viscosity of poly-L-lactic acid solutions with concentration of $4 \% \mathrm{w} / \mathrm{w}, 6 \% \mathrm{w} / \mathrm{w}$, and $8 \%$ $w / \mathrm{w}$ was $52 \pm 1 \mathrm{mPa} \cdot \mathrm{s}, 347 \pm 39 \mathrm{mPa} \cdot \mathrm{s}$, and $2574 \pm 658 \mathrm{mPa} \cdot \mathrm{s}$, respectively.

\section{Multi-jet electrospinning of poly-L-lactic acid nanofibers}

The multi-jet electrospun (MES) nanofibrous mats were prepared in the system described above (see Fig. 1-a and b). Polymer solution was delivered to the system by syringe pump with constant feed rate of $15 \mathrm{ml} \cdot \mathrm{h}^{-1}$, about tenfold higher than reported for classic electrospinning [2]. The applied high voltage of 15,20 and $25 \mathrm{kV}$ generated the electrostatic field causing stretching of the poly-L-lactic acid solutions and formation of nanofibers by solvent evaporation. Resulting nanofibers were collected on the surface of a grounded aluminum plate. The MES process was conducted in the room temperature, and relative humidity was in a range between 40 to $55 \%$, conditions corresponding to preparation of the polymer solutions.

\section{Blow-assisted multi-jet electrospinning of poly-L-lactic acid nanofibers}

The blow-assisted multi-jet electrospun (BMES) nanofibrous mats were produced in the system described above (Fig. 1), with blowing assistance by high speed air stream (Fig. 1c). The procedure of BMES, feed rate and voltages were the same as for the multi-jet electrospinning (MES) process, however the compressed air stream was applied as an assistance for the process. Using air pump and reductive valves system, the air stream with inlet pressure of 3 bars was used. As well as MES, the BMES was conducted in the room temperature, and relative humidity was in a range between 40 to $55 \%$, conditions corresponding to preparation of the polymer solutions.

\section{Nanofibers deposition analysis}

The collecting patterns and the size of nanofibrous mats produced in multi-jet electrospinning (MES) and blow-assisted multi-jet electrospinning (BMES) on aluminum collecting plate were investigated. Both processes were conducted for $5 \mathrm{~min}$. After that, the collector was removed from the system to take a photograph. For products from both processes - MES 
and BMES - the area of the collector covered by the nanofibrous mat was measured using ImageJ software (version 2.0.0-rc-43/1.50 g) for the comparison.

\section{Scanning electron microscopy analysis}

The differences in morphology and the size between poly-Llactic acid (PLLA) nanofibers obtained in multi-jet electrospinning (MES) and blow-assisted multi-jet electrospinning (BMES) were determined based on the analysis of scanning electron microscope (SEM, PhenomTM, Phenom World, Netherlands) micrographs. Nanofibrous mats were cut into square pieces $10 \mathrm{~mm} \times 10 \mathrm{~mm}$ and sputtered with gold (K550 Emitech, Quorum Technologies, UK) prior SEM analysis. ImageJ software (version 2.0.0-rc-43/1.50 g) was used to measure the fibers diameter on the SEM micrographs.

\section{Statistics}

Results from experiments of collecting of nanofibrous mats are expressed as a mean value of the nanofibers mat area \pm standard error (SE). Experiments were performed five times for each processing conditions. For each experiment, the total area of the nanofibrous mat was measured. Results of nanofibers mean diameter analysis are expressed as mean value \pm standard error (SE). One hundred diameters of single fiber were measured for each experiment to calculate mean nanofiber diameter value. Experiments of nanofibers mean diameter dependence on the applied voltage, as well as viscosity measurements are expressed as mean value \pm standard deviation (SD) calculated based on experiments repeated five times. Statistical significance of difference between compared populations was analyzed using single factor analysis of variance (one-way ANOVA) with $p<0.05$ and the results of the analysis are denoted in figures with asterisk.

\section{Results}

\section{Nanofibers deposition analysis}

Mutual electrostatic repulsion of polymeric jets in multi-jet electrospinning (MES) is one of the main drawbacks of this technique. It affects the stability of the process and uniformity of produced nanofibrous mats, as well as the area of the collector covered by the product in the process, which can generate product loss. In Fig. 2 we present data that describes the difference between collecting the nanofibers in MES and BMES. The area of the nanofibrous mat collected in the blow-assisted multi-jet electrospun process is from about $33 \%$ to about $42 \%$ lower comparing to the product of the multi-jet electrospinning process. What is more, the area of the nanofibrous mat increases with the increase of the polymer solution concentration, regardless of the process.

\section{Nanofibers morphology and sizes}

The key properties of the nanofibrous mats are uniform fibers morphology and narrow size distribution within the single
Fig. 2 Comparison of collecting of nanofibers in multi-jet electrospinning system (MES) and blow-assisted multi-jet electrospinning system (BMES) for poly-L-lactic acid solutions (PLLA), $\mathrm{V}=20 \mathrm{kV}, p=3$ bar (in case of BMES); the asterisk denotes significant difference $(p<0.05)$ between area of nanofibrous mat from MES and BMES

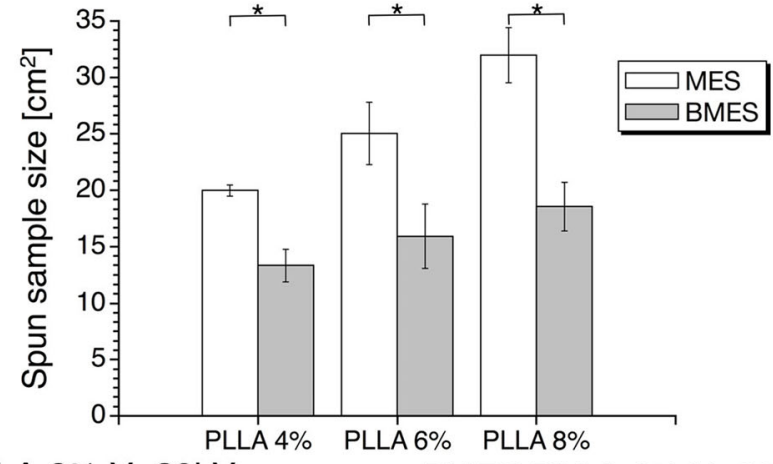

MES PLLA $6 \% \mathrm{~V}=20 \mathrm{kV}$ BMES PLLA $6 \% \mathrm{~V}=20 \mathrm{kV} p=3$ bar

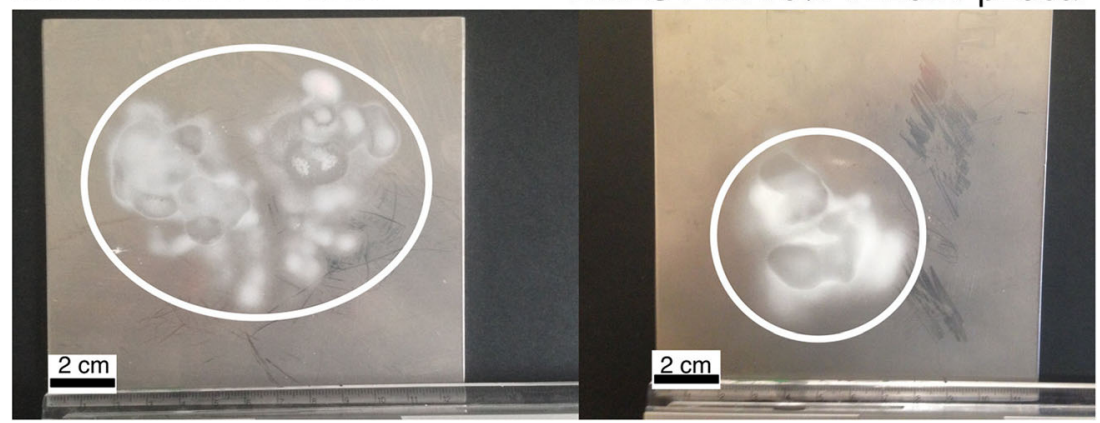


mat, when mean fiber diameter is the most widely used as a characterization parameter for the product. Proposing more efficient technology for the nanofibers production, one needs to remember that the efficiency of a technology must be in parallel to the product quality. Figure 3 shows results of scanning electron microscope (SEM) analysis of multi-jet electrospun (MES) and blow-assisted multi-jet electrospun (BMES) nanofibers spun from poly-L-lactic acid (PLLA) solution with concentration of $4 \% \mathrm{w} / \mathrm{w}$. Regardless of the production process, nanofibers contain beads within their structure. BMES process allows nanofibers formation for all of applied voltage values, from 15 to $25 \mathrm{kV}$, comparing to MES process which produces PLLA foil when $20 \mathrm{kV}$ voltage was applied. Even though the product exhibit beaded morphology, if we take only nanofibers into account they show narrow size distributions, where mean fiber diameter is in a range from about $150 \mathrm{~nm}$ to about $180 \mathrm{~nm}$ for multi-jet electrospinning process, and from about $140 \mathrm{~nm}$ to about $170 \mathrm{~nm}$ for blow-assisted multi-jet electrospun, when the air pressure is 3 bars.

Nanofibers produced from PLLA solution with $6 \% \mathrm{w} / \mathrm{w}$ concentration of polymer have less beads within the structure comparing to the nanofibers produced from $4 \% \mathrm{w} / \mathrm{w}$ solution, see Fig. 4. In the same manner as in electrospinning, MES nanofibers spun from solution with increasing polymer concentration exhibit finer fibers morphology maintaining narrow size distribution [33]. Application of blow-assistance to the multi-jet process results with nanofibers with fewer and more stretched beads in fiber structure, as shown in SEM micrographs (Fig. 4). Nanofibers size distributions for MES are much narrower than for BMES. Mean fiber diameter for multi-jet electrospinning nanofibers ranges from about $130 \mathrm{~nm}$ for $15 \mathrm{kV}$ voltage to about $280 \mathrm{~nm}$ for $25 \mathrm{kV}$, when application of air blowing-assistance with a pressure of 3 bars in blow-assisted multi-jet electrospinning results with product with mean fiber diameter from about $230 \mathrm{~nm}$ to about $330 \mathrm{~nm}$.

Figure 5 shows morphology of nanofibers produced in multi-jet electrospinning and blow-assisted multi-jet electrospinning from poly-L-lactic acid solution with $8 \% \mathrm{w} / \mathrm{w}$ polymer concentration. Application of high electrostatic voltage of $20 \mathrm{kV}$ and $25 \mathrm{kV}$ in MES process results with fine nanofibers, while $15 \mathrm{kV}$ causes polymer solution to create a foil instead of nanofibers. What is more, application of BMES with air pressure of 3 bars allows production of fine nanofibers, in the range of voltages from $15 \mathrm{kV}$ to $25 \mathrm{kV}$, with relatively narrow size distribution for thicker fibers. Mean fiber diameter for multi-jet electrospinning ranges from about $700 \mathrm{~nm}$ to about $850 \mathrm{~nm}$, and for blow-assisted multi-jet electrospinning - from about $700 \mathrm{~nm}$ to about $900 \mathrm{~nm}$.
Fig. 3 SEM micrographs of multi-jet electrospinning system (MES) and blow-assisted multijet electrospinning system (BMES) nanofibers; normal size distribution of MES and BMES nanofibers; process parameters and mean fiber diameter of nanofibers spun from poly-Llactic acid (PLLA) solution with concentration of $4 \% \mathrm{w} / \mathrm{w}$ - note that sample MES PLLA 4\% $\mathrm{V}=20 \mathrm{kV}$ contains only a few fibers and was not considered in size distribution and mean value calculations

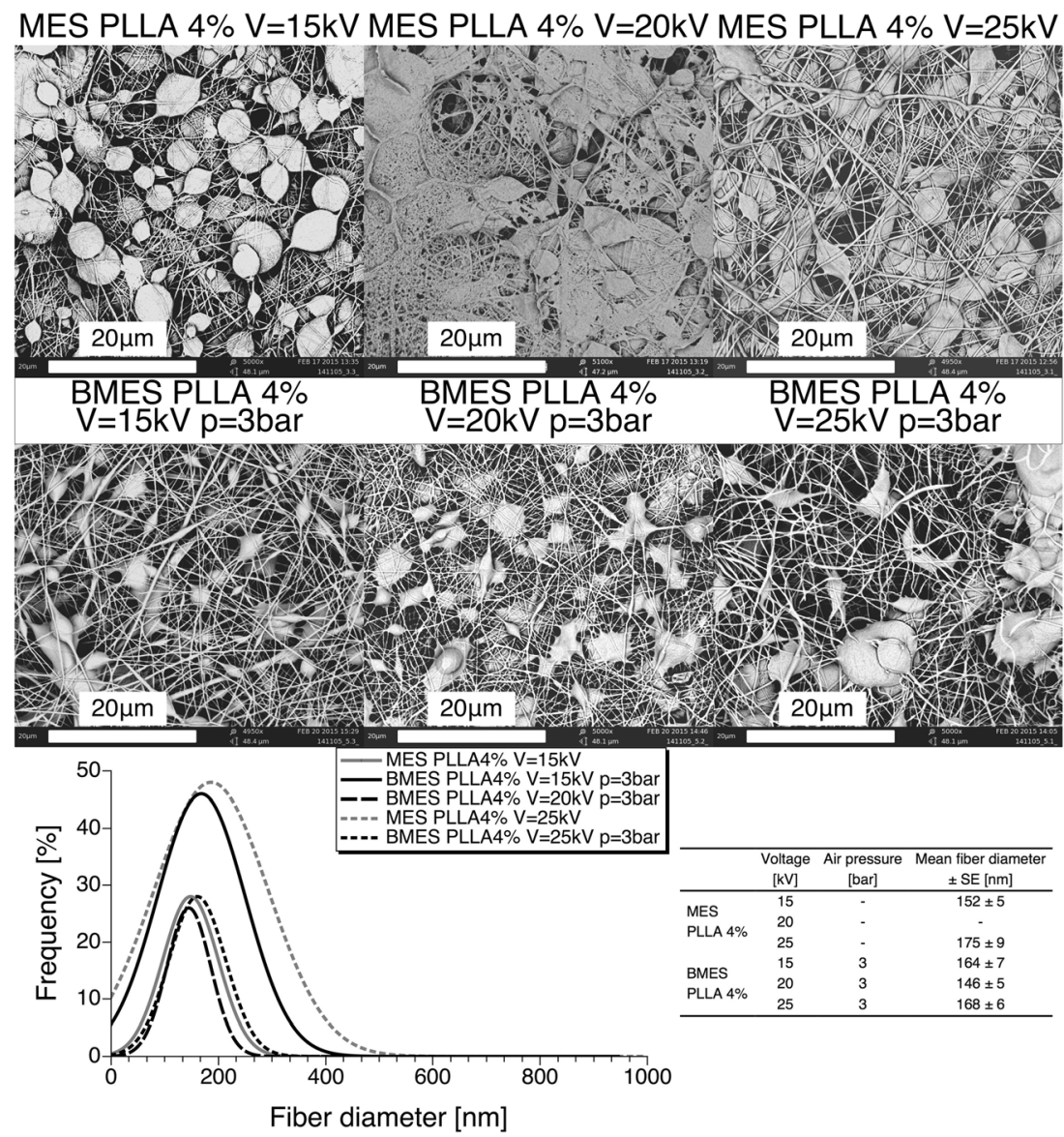


Fig. 4 SEM micrographs of multi-jet electrospinning system (MES) and blow-assisted multijet electrospinning system (BMES) nanofibers; normal size distribution of MES and BMES nanofibers; process parameters and mean fiber diameter of nanofibers spun from poly-Llactic acid (PLLA) solution with concentration of $6 \% \mathrm{w} / \mathrm{w}$
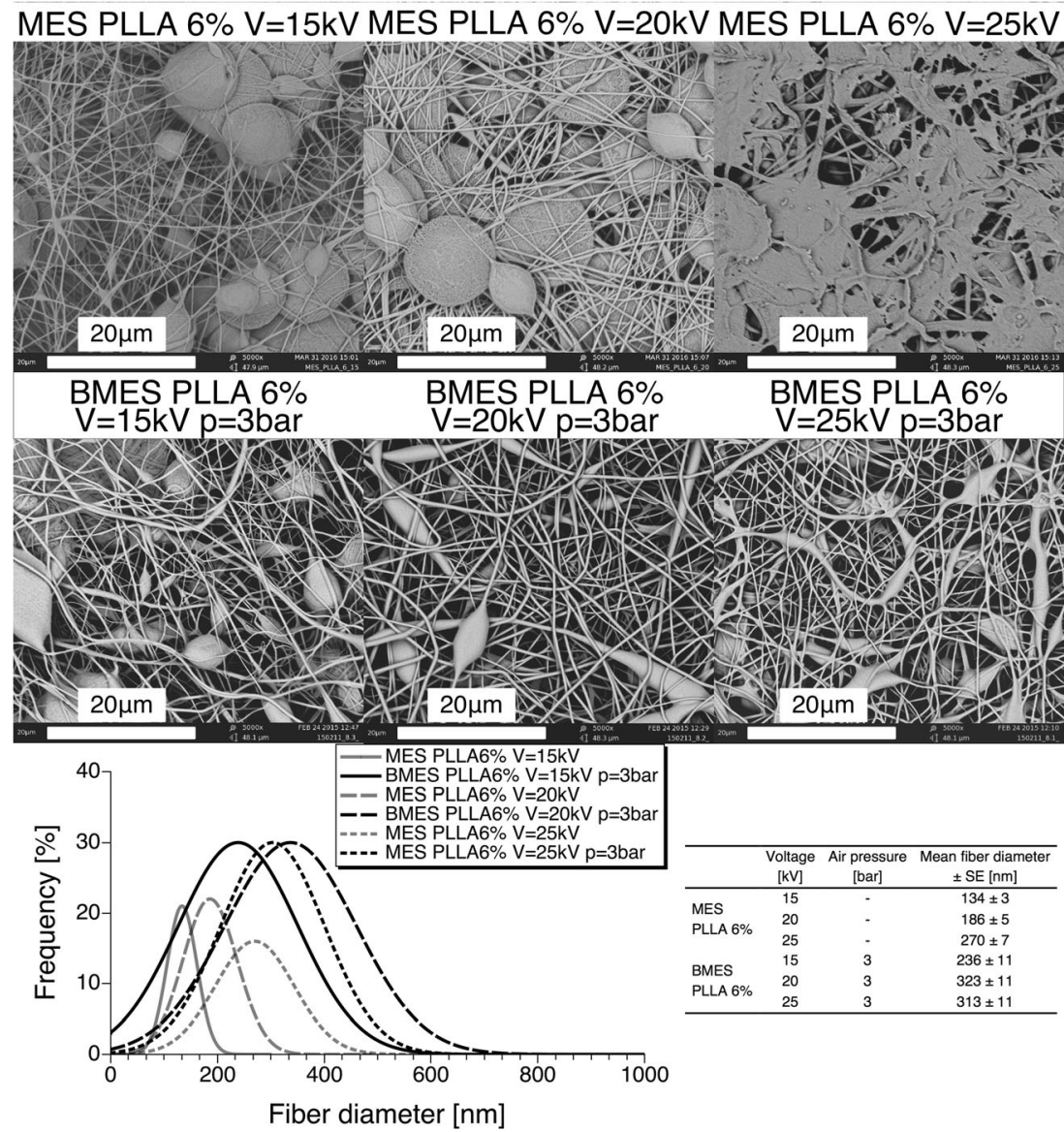

We observed that changes in applied voltage in both, multijet electrospinning and blow-assisted multi-jet electrospinning processes influences final product's morphology and mean fiber diameter. Figure 6 presents data indicating that in case of multi-jet electrospinning process for PLLA solution with $6 \% \mathrm{w} / \mathrm{w}$ polymer concentration mean fiber diameter increases with voltage. Mean fiber diameter reaches maximum value (in the investigated range of voltages) for PLLA solutions with polymer concentration of $6 \% \mathrm{w} / \mathrm{w}$ and $8 \% \mathrm{w} / \mathrm{w}$ in blowassisted multi-jet electrospinning for $20 \mathrm{kV}$. However, for PLLA solution with $4 \% \mathrm{w} / \mathrm{w}$ polymer concentration spun in blow-assisted multi-jet electrospinning process increase of voltage does not affect the mean fiber diameter.

\section{Discussion}

Recently reported modifications of multi-jet electrospinning (MES) systems in order to overcome the mutual repulsion effect of polymer solution jets include application of additional electrodes, auxiliary electrodes and specifically designed polymer nozzles arrays [21, 24, 34]. Most of those approaches result in the complex design of the system and/or complex modeling of electrostatic field and flow patterns in order to optimize the nozzles array of the multi-jet electrospinning system. Application of air blow-assistance to the multi-jet electrospinning (BMES) allows simple and precise control of nanofibers collecting process, by the reduction of the collecting area. The viscous interaction between air stream and polymer solution jets in blow-assisted multi-jet electrospinning lowers the deviation of jet angles described by Zheng et al. and reduces the envelope cones of the jets [3]. This leads to the reduction of the collecting area of the nanofibrous mat resulting with more uniform product. However, the air stream affects the nanofibers formation process, thus proper optimization of processing parameters (voltage, air pressure, and the like) might be necessary to produce fibers without defects like beads or foil on the surface of the nanofibrous mat.

In electrospinning the effect of beads formation is wellknown [33]. Low viscosity of polymer solution and high surface tension of the solution cause instabilities in classic singlejet electrospinning. This leads to beads formation within the fibers structure $[35,36]$. We observed the same effect in both, multi-jet electrospinning (MES) and blow-assisted multi-jet electrospinning (BMES). MES and BMES processes conducted with poly-L-lactic acid (PLLA) solutions with concentration of $4 \% \mathrm{w} / \mathrm{w}$ and $6 \% \mathrm{w} / \mathrm{w}$ - relatively low polymer concentration in solutions and low solutions viscosity - results with beaded nanofibers. Spinning with PLLA solution with 
Fig. 5 SEM micrographs of multi-jet electrospinning system (MES) and blow-assisted multijet electrospinning system (BMES) nanofibers; normal size distribution of MES and BMES nanofibers; process parameters and mean fiber diameter of nanofibers spun from poly-Llactic acid (PLLA) solution with concentration of $8 \% \mathrm{w} / \mathrm{w}$ - note that sample MES PLLA $8 \%$ $\mathrm{V}=15 \mathrm{kV}$ exhibit foil morphology and was not considered in size distribution and mean value calculations
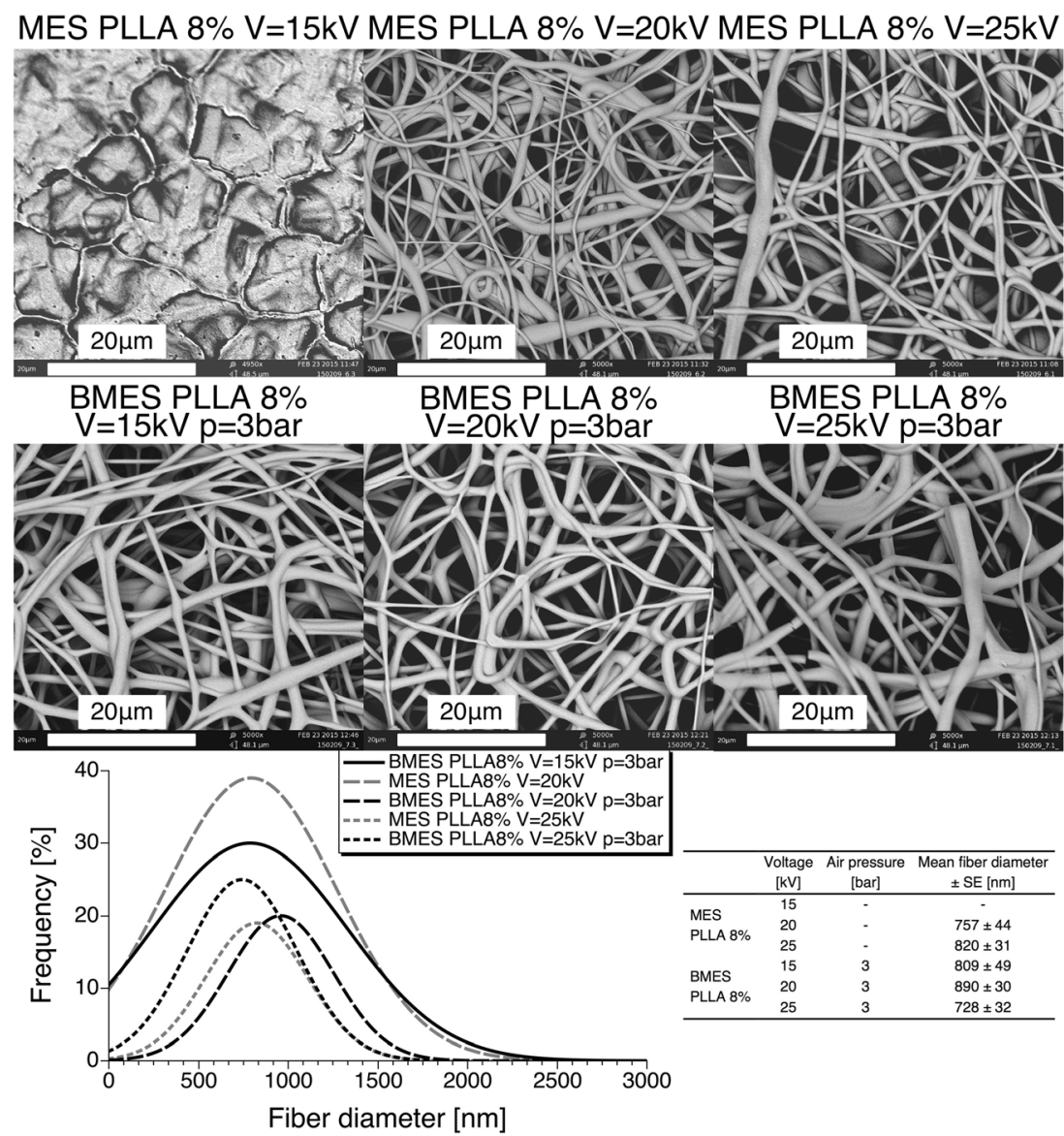

polymer concentration of $8 \% \mathrm{w} / \mathrm{w}$ allows production of beadfree nanofibers, regardless of the spinning process.

Increasing the polymer solution concentration (and viscosity) leads not only to the formation of bead-free nanofibers in most spinning processes (like electrospinning or solution blow spinning). It also leads to the increase of mean fiber diameter and the slight broadening of a fibers size distribution [32]. This effect appeared in both investigated processes: multi-jet

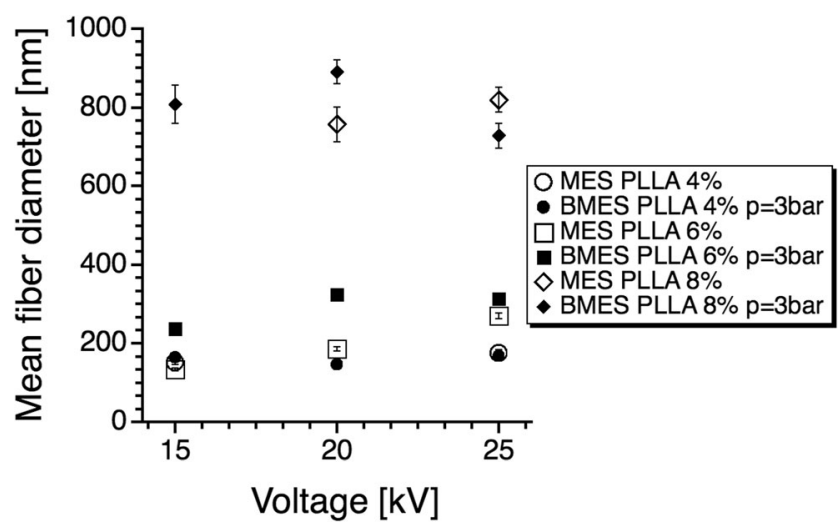

Fig. 6 The change in the mean fiber diameter for poly-L-lactic acid (PLLA) nanofibers produced in multi-jet electrospinning system (MES) and blow-assisted multi-jet electrospinning system (BMES) with the applied voltage electrospinning and blow-assisted multi-jet electrospinning. Nanofibers obtained in BMES exhibit the mean fiber diameter from about $50 \mathrm{~nm}$ to $100 \mathrm{~nm}$ higher comparing to nanofibers from MES process with the same applied voltage (for PLLA solutions with concentration of $6 \% \mathrm{w} / \mathrm{w}$ and $8 \% \mathrm{w} / \mathrm{w}$ ). This difference between mean fiber diameter obtained in multi-jet electrospinning process and blow-assisted process has also a different cause than influence of the polymer solution properties. The main driving force of the nanofibers formation in MES process is electrostatic force - generated between the positively charged polymer nozzles electrode and the grounded collector - acting on the polymer solution jet. The polymer jet charged in this manner interacts not only with a collector, jet itself (causing bending instabilities), but also with other polymer jets in MES. Mutual electrostatic repulsion causes broadening of nanofibers collecting area in this process, because of the deviations of the jet angle in the process. All of these interactions of polymeric jets in multi-jet electrospinning process effect with increase of the distance the polymer jets needs to go through from polymer nozzles to the collector surface. This gives a polymer jets longer time to stretch and generate thin nanofibers, similar to the effects reported elsewhere for single-jet electrospinning [35]. In blow-assisted multi-jet electrospinning, the electrostatic force of the nanofibers formation is not the only driving force for the process. 
There are additional viscous interactions of high speed air surrounding polymer jets in this process. What is more, the same viscous interactions between polymer jets and air stream reduces deviation of the polymer jets angle. This results with reduction of mutual electrostatic repulsion effect acting on polymer jets, thus reduction of bending instabilities of polymer jets, and further - reduction of flight time of the polymer jets from the nozzles to collecting plate. Shorter the flight time of polymer jet in the nanofibers formation process, shorter the time for stretching the jet. Those lead to the generation of the thicker resulting nanofibers in blow-assisted multi-jet electrospinning process.

\section{Conclusions}

In this work, we report design and development of the multi-jet electrospinning (MES) system, with additional blow-assistance (BMES) for poly-L-lactic acid (PLLA) nanofibers production. The system allows to overcome the mutual electrostatic repulsion issue in multi-jet electrospinning by using sheath gas stream for the process. The reduction of the deviation of polymer jets in the process leads to reduction of the area of nanofibrous mat by about 30 to $40 \%$, reducing instability problems such as dripping of the polymer solution and fiber collection difficulties. Additionally, system allows nanofibers formation from PLLA solutions with concentration of polymer from $4 \% \mathrm{w} / \mathrm{w}$, through $6 \% \mathrm{w} / \mathrm{w}$, to $8 \% \mathrm{w} / \mathrm{w}$ - from solutions with low viscosity to high viscosity. Resulting nanofibers exhibit mean fiber diameter in the range from about $140 \mathrm{~nm}$ to about $180 \mathrm{~nm}(4 \% \mathrm{w} / \mathrm{w}$ poly-Llactic acid concentration), from about $130 \mathrm{~nm}$ to about $330 \mathrm{~nm}$ (6\% $\mathrm{w} / \mathrm{w}$ poly-L-lactic acid concentration), and from about $700 \mathrm{~nm}$ to about $900 \mathrm{~nm}(8 \%$ w/w poly-L-lactic acid concentration), respectively, for both investigated modes - multi-jet electrospinning and blow-assisted multi-jet electrospinning. Using multi-jet approach to electrospinning improves the process throughput and application of blow-assistance decreases the product loss in multi-jet spinning process. Proposed system, with further improvement of the design and with proper processing parameters optimization, might be the next step towards the scale up of electrospinning of polymer nanofibers.

Acknowledgements Authors would like to acknowledge an invaluable help of Andrzej Krasiński, PhD, and Faculty of Chemical and Process Engineering Workshop with manufacturing the blow-assisted multi-jet electrospinning system prototype.

Open Access This article is distributed under the terms of the Creative Commons Attribution 4.0 International License (http:// creativecommons.org/licenses/by/4.0/), which permits unrestricted use, distribution, and reproduction in any medium, provided you give appropriate credit to the original author(s) and the source, provide a link to the Creative Commons license, and indicate if changes were made.

\section{References}

1. Formhals A (1934) US Patent 1:975,504

2. Borrotti M, Lanzarone E, Manganini F, et al (2017) Defect minimization and feature control in electrospinning through design of experiments. J Appl Polym Sci 134:333-310. doi:10.1002/app.44740

3. Zheng Y, Gong RH, Zeng Y (2015) Multijet motion and deviation in electrospinning. RSC Adv 5:48533-48540. doi:10.1039/C5RA06049D

4. Gopal R, Kaur S, Ma Z, et al (2006) Electrospun nanofibrous filtration membrane. J Membr Sci 281:581-586. doi:10.1016/j. memsci.2006.04.026

5. Zhang S, Liu H, Yin X, et al (2016) Anti-deformed Polyacrylonitrile/Polysulfone composite membrane with binary structures for effective air filtration. ACS Appl Mater Interfaces 8:8086-8095. doi:10.1021/acsami.6b00359

6. Chen S, Hou H, Harnisch F, et al (2011) Electrospun and solution blown three-dimensional carbon fiber nonwovens for application as electrodes in microbial fuel cells. Energy Environ Sci 4:1417-1421. doi:10.1039/c0ee00446d

7. Kumar B, Asadi M, Pisasale D, et al (2013) Renewable and metalfree carbon nanofibre catalysts for carbon dioxide reduction. Nat Commun 4:1-8. doi:10.1038/ncomms3819

8. Li Z, Zhang J-W, Yu L-G, Zhang J-W (2017) Electrospun porous nanofibers for electrochemical energy storage. J Mater Sci 52: 6173-6195. doi:10.1007/s10853-017-0794-2

9. Thenmozhi S, Dharmaraj N, Kadirvelu K, Kim HY (2017) Electrospun nanofibers: new generation materials for advanced applications. Mater Sci Eng B-Adv 217:36-48. doi:10.1016/j.mseb.2017.01.001

10. Sakai S, Yamada Y, Yamaguchi T, et al (2008) Surface immobilization of poly(ethyleneimine) and plasmid DNA on electrospun poly(L-lactic acid) fibrous mats using a layer-bylayer approach for gene delivery. J Biomed Mater Res A 88A: 281-287. doi:10.1002/jbm.a.31870

11. Pilarek M, Grabowska I, Senderek I, et al (2014) Liquid perfluorochemical-supported hybrid cell culture system for proliferation of chondrocytes on fibrous polylactide scaffolds. Bioprocess Biosyst Eng 37:1707-1715. doi:10.1007/s00449014-1143-3

12. Kitsara M, Agbulut O, Kontziampasis D, et al (2017) Fibers for hearts: a critical review on electrospinning for cardiac tissue engineering. Acta Biomater 48:20-40. doi:10.1016/j.actbio.2016.11.014

13. Zheng Y, Zhuang C, Gong RH, Zeng Y (2014) Electric field Design for Multijet Electropsinning with uniform electric field. Ind Eng Chem Res 53:14876-14884. doi:10.1021/ie501827b

14. Theron SA, Yarin AL, Zussman E, Kroll E (2005) Multiple jets in electrospinning: experiment and modeling. Polymer 46:28892899. doi:10.1016/j.polymer.2005.01.054

15. Xie S, Zeng Y (2012) Effects of electric field on Multineedle electrospinning: experiment and simulation study. Ind Eng Chem Res 51:5336-5345. doi:10.1021/ie2020763

16. Zhou F-L, Gong R-H, Porat I (2009) Mass production of nanofibre assemblies by electrostatic spinning. Polym Int 58: 331-342. doi:10.1002/pi.2521

17. Varabhas JS, Chase GG, Reneker DH (2008) Electrospun nanofibers from a porous hollow tube. Polymer 49:42264229. doi:10.1016/j.polymer.2008.07.043

18. Forward KM, Rutledge GC (2012) Free surface electrospinning from a wire electrode. Chem Eng J 183:492-503. doi:10.1016/j. cej.2011.12.045

19. Jiang G, Zhang S, Qin X (2013) High throughput of quality nanofibers via one stepped pyramid-shaped spinneret. Mater Lett 106: 56-58. doi:10.1016/j.matlet.2013.04.084 
20. Deitzel JM, Kleinmeyer JD, Hirvonen JK, Tan NCB (2001) Controlled deposition of electrospun poly (ethylene oxide) fibers. Polymer 42:8163-8170

21. Kim G, Cho Y-S, Kim WD (2006) Stability analysis for multi-jets electrospinning process modified with a cylindrical electrode. Eur Polym J 42:2031-2038. doi:10.1016/j.eurpolymj.2006.01.026

22. Angammana CJ, Jayaram SH (2011) The effects of electric field on the Multijet electrospinning process and fiber morphology. IEEE T Ind Appl 47:1028-1035

23. Qiu Y, Yu J, Rafique J, et al (2009) Large-scale production of aligned long boron nitride Nanofibers by Multijet/ Multicollector electrospinning. J Phys Chem C 113:1122811234. doi:10.1021/jp901267k

24. Tomaszewski W, Szadkowski M (2005) Investigation of electrospinning with the use of a multi-jet electrospinning head. Fibres Text East Eur 13:22-26

25. Yang Y, Jia Z, Li Q, et al (2010) A shield ring enhanced equilateral hexagon distributed multi-needle electrospinning spinneret. IEEE T Dielect El In 17:1592-1601. doi:10.1109/TDEI.2010.5595562

26. Ramakrishnan R, Gimbun J, Samsuri F, et al (2016) Needleless electrospinning technology - an entrepreneurial perspective. Indian J Sci Technol 9:1-11. doi:10.17485/ijst/2016/v9i15/91538

27. Wang X, Um IC, Fang D, et al (2005) Formation of waterresistant hyaluronic acid nanofibers by blowing-assisted electro-spinning and non-toxic post treatments. Polymer 46: 4853-4867. doi:10.1016/j.polymer.2005.03.058

28. He J, Lian Y, Zhang X, et al (2014) Mass preparation of nanofibers by high pressure air-jet split electrospinning: effect of electric field. J Polym Sci Pol Phys 52:993-1001. doi:10.1002/polb.23519
29. Pu C, He J, Cui S, Gao W (2014) Fabrication of nanofibers by a modified air-jet electrospinning method. Iran Polym J 23:13-25. doi:10.1007/s13726-013-0195-6

30. Yu Z, Lin Y, Huang W et al (2014) Multi spinnerets electrospinning with assistant sheath gas. Proceedings of the IEEE international conference on NanoMicro engineered and molecular systems 64-67

31. Medeiros ES, Glenn GM, Klamczynski AP, et al (2009) Solution blow spinning: a new method to produce micro- and Nanofibers from polymer solutions. J Appl Polym Sci 113: 2322-2330. doi:10.1002/app.30275

32. Wojasiński M, Pilarek M, Ciach T (2014) Comparative studies of electrospinning and solution blow spinning processes for the production of nanofibrous poly(L-lactic acid) materials for biomedical engineering. Pol J Chem Technol 16:43-50. doi:10.2478/pjct-2014-0028

33. Lee KH, Kim HY, Bang HJ, et al (2003) The change of bead morphology formed on electrospun polystyrene fibers. Polymer 44: 4029-4034. doi:10.1016/S0032-3861(03)00345-8

34. Kumar A, Wei M, Barry C, et al (2010) Controlling fiber repulsion in Multijet electrospinning for higher throughput. Macromol Mater Eng 295:701-708. doi:10.1002/mame.200900425

35. Reneker DH, Yarin AL, Fong H, Koombhongse S (2000) Bending instability of electrically charged liquid jets of polymer solutions in electrospinning. J Appl Phys 87:4531-4518. doi:10.1063/1.373532

36. Yarin AL, Koombhongse S, Reneker DH (2001) Bending instability in electrospinning of nanofibers. J Appl Phys 89:3018-3026. doi:10.1063/1.1333035 